\title{
PRIMER REGISTRO DE MEPHITIDAE (CARNIVORA: MAMMALIA) PARA EL PLEISTOCENO DE CHILE
}

\author{
ERWIN GONZÁLEZ G., FRANCISCO J. PREVOSTI" Y MARIO PINO Q."
}

\begin{abstract}
RESUMEN
Damos a conocer el primer registro fósil de la familia Mephitidae para el Pleistoceno de Chile, el cual corresponde a gran parte de la caja craneana. El material fue descubierto en el sitio paleontológico Pilauco, Osorno, Chile (40³9'S-7307’ W) cuyos sedimentos fueron depositados durante el Pleistoceno tardío (Edad Lujanense). Los registros previos de Mephitidae en Sudamérica, fósiles y actuales, han sido incluidos en el género Conepatus Gray, 1837. El registro fósil que se ha publicado, proviene de sitios paleontológicos de Argentina, Bolivia, Brasil, Perú y Venezuela, cubriendo gran parte de su distribución en el presente. Sin embargo, existen muchas áreas sin registros, incluyendo a Chile. En la presente investigación comparamos medidas del cráneo de ejemplares actuales y fósiles de la familia. Nuestros resultados indicaron que la caja craneana corresponde a Mephitidae, y específicamente al género Conepatus. Además, se amplía la paleodistribución del género, siendo este el primer registro fósil para el Pleistoceno de Chile.
\end{abstract}

PALABRAS CLAVES: Conepatus, Mephitidae, Pilauco, Pleistoceno, Chile.

\section{FIRST RECORD OF MEPHITIDAE (CARNIVORA: MAMMALIA) FOR THE PLEISTOCENE OF CHILE}

\begin{abstract}
We report the first fossil record of the Mephitidae family in Chile, which consists of a large portion of the cranium. The material was discovered in the Pilauco paleontological site, Osorno, Chile (40³9'S$73^{\circ} 07^{\prime} \mathrm{W}$ ) and was deposited during the late Pleistocene (Lujanense age). The previous records of fossil and present day South American Mephitidae have been included in the genus Conepatus Gray, 1837. The published fossil record comes from paleontological sites in Argentina, Bolivia, Brasil, Perú and Venezuela, which cover most of the present distribution. However, there are many areas that lack records,

Laboratorio de Paleoecología, Instituto de Geociencias, Facultad de Ciencias. Campus Isla Teja, Universidad Austral de Chile, Casilla 567 Valdivia, Chile.e.gonzalezguarda@gmail.com; mariopino@uach.cl

* División Mastozoológicas Museo Argentino de Ciencias Naturales "Bernardino Rivadavia”-CONICET. Avenida Ángel Gallardo 470 C1405DJR-Buenos Aires-Argentina. protocyon@hotmail.com
\end{abstract}


such as Chile. Using the comparative method based on cranium measures and applied to present and fossil specimens of the above mentioned family, our results show that the discovered cranium belongs to Mephitidae and specifically to the genus Conepatus. Furthermore, the paleodistribution of the genus is extended with the first Pleistocene fossil record for Chile.

KEYWORDS: Conepatus, Mephitidae, Pilauco, Pleistocene, Chile.

\section{INTRODUCCIÓN}

Históricamente, Mephitinae ha sido considerada una subfamilia de Mustelidae, pero recientes filogenias han mostrado que Mephitinae es el grupo hermano de Mustelidae y Procyonidae, por lo que ha sido elevada al nivel de familia. (Dragoo y Honeucutt 1997; Sato et al. 2004; Flynn et al. 2005a). Conepatus es el único género con especies vivientes en Sudamérica, el cual incluye C. chinga Molina, 1782 y C. humboldtii Gray, 1837, dos especies restringidas a este continente, y C. semistriatus Boddaert, 1784 que se distribuye desde Centroamérica hasta el norte de Sudamérica (Dragoo et al. 2003). En
Chile, C. chinga se encuentra en la Región de Arica y Parinacota y en la Región de Coquimbo hasta los $40^{\circ} \mathrm{S}$ (Quintana et al. 2000) y en Argentina ocupa el norte y centro hasta los $42^{\circ} \mathrm{S}$ (Parera, 2002). $C$. humboldtii, se distribuye en Chile desde los $39^{\circ} \mathrm{S}$ y en Argentina desde los $38^{\circ}-42^{\circ} \mathrm{S}$ hasta el Estrecho de Magallanes (Fuller et al. 1987). Según las últimas revisiones (Berman, 1994; Forasiepi, 2003) sólo Conepatus ha inmigrado a América del Sur y la inclusión de algunos fósiles en Mephitis É. Geoffroy Saint-Hilaire y Cuvier, 1795 estarían basados en arreglos taxonómicos desactualizados o erróneos. Restos fósiles de Conepatus se han registrado en Venezuela, Perú, Bolivia, Argentina y Brasil (Figura

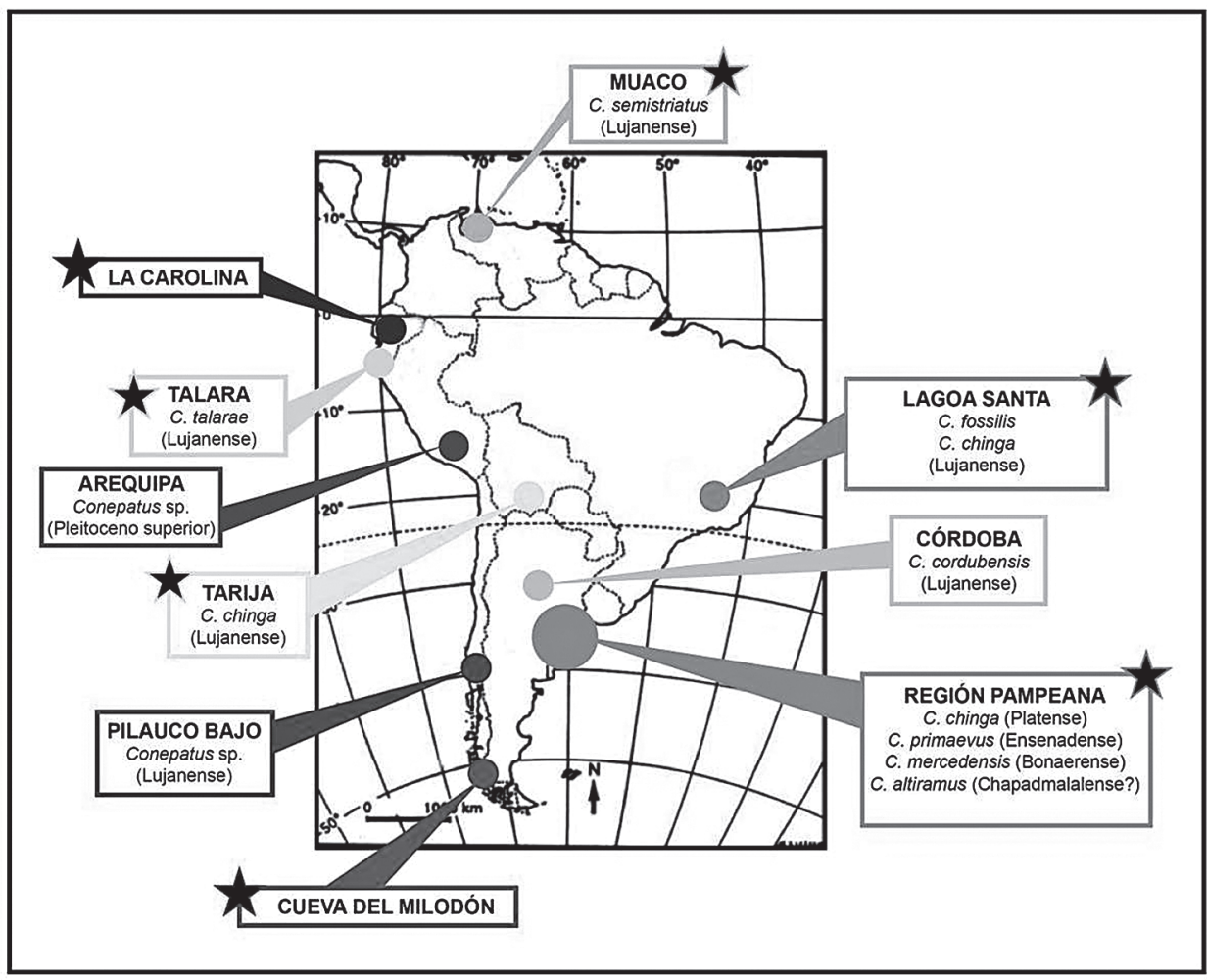

Fig. 1. Mapa de la distribución de los registros fósiles de Conepatus que se han reconocido en la literatura para América del Sur. Las principales localidades fosilíferas del Plioceno y Cuaternario de América del Sur se hacen notar con estrellas. 


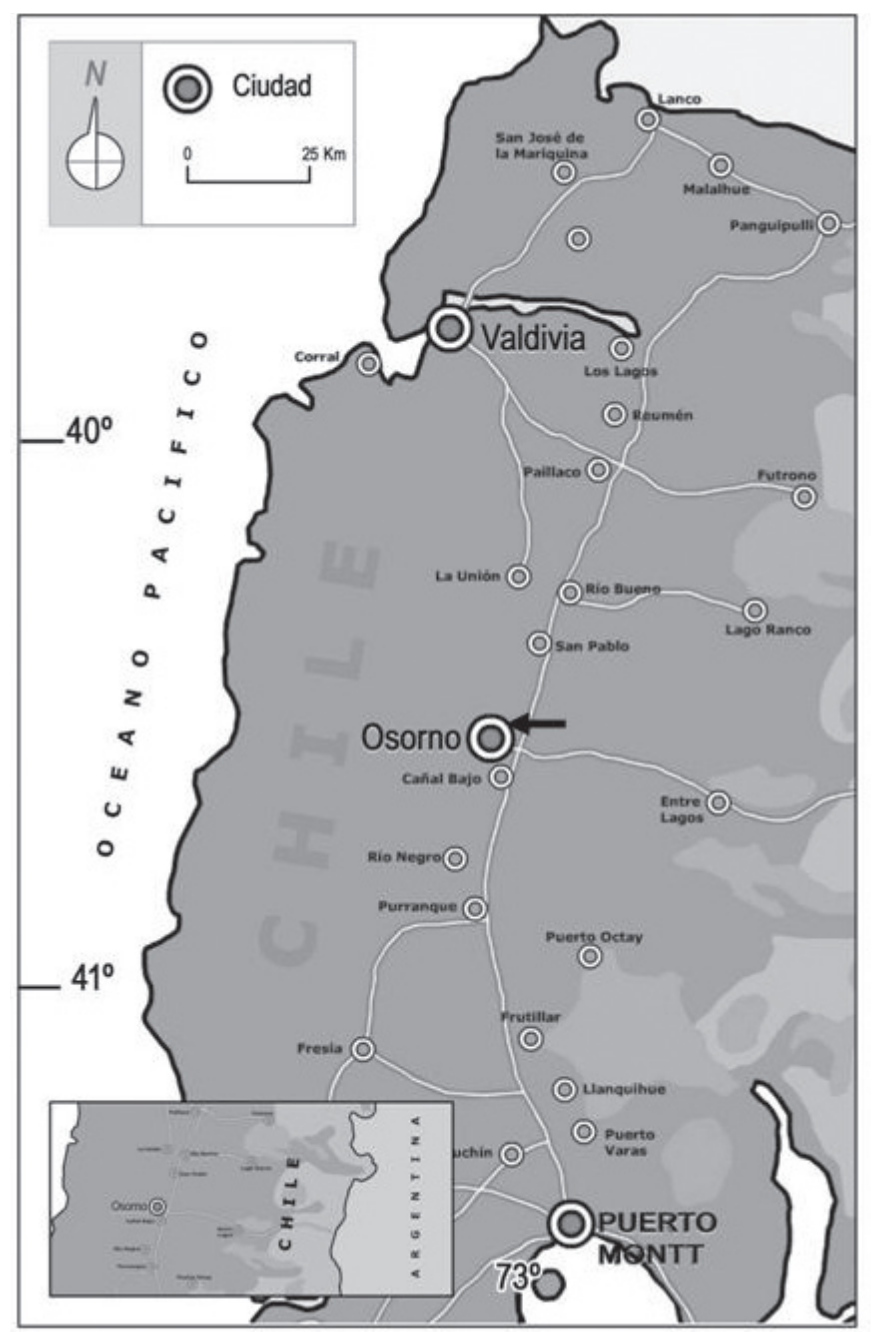

Fig. 2. Ubicación del Sitio Paleontológico Pilauco. Es indicada por la flecha negra.

1; Bocquentin-Villanueva, 1979; Churcher y Van Zyll de Jong, 1965; Hoffstetter, 1963; Ameghino, 1889; y Paula Couto, 1979). Wang y CarranzaCastañeda (2008) afirman que C. altiramus Reig, 1952 proviene del Plioceno tardío, aunque no aportan datos relevantes sobre su procedencia que permitan dilucidar las dudas existentes sobre la ubicación geográfica de esta especie (e.g Churcher y Zyll de Jong, 1965; Berman, 1994) por lo que no se puede descartar que provenga de niveles más modernos (Marplatense-Lujanense; Berman 1994). Sin tener en cuenta a esta especie, los registros más antiguos de méfitidos provienen del Ensenadense (Pleistoceno temprano-medio) (Berman, 1994; Woodburne et al. 2006; Soibelzon y Prevosti, 2007). La presente investigación tiene como principal objetivo dar a conocer el primer registro de Mephitidae para el Pleistoceno de Chile, el cual corresponde a una caja craneana encontrada en el Sitio Paleontológico Pilauco, Osorno, Chile (Figura 2).

Abreviaturas de instituciones MHMO, Museo Histórico Municipal de Osorno; LPUACh, Laboratorio de Paleoecología de la Universidad Austral de Chile; MACN, Museo Argentino de Ciencias Naturales; MLP, Museo de la Plata; UCV, Universidad Central de Venezuela; IML, Instituto Miguel Lillo; MPS, Museo Paleontológico Municipal de San Pedro "Fray Manuel Torres"; NMW; Naturhistorisches Museum Wien. 


\section{MATERIALES Y MÉTODOS}

El fósil estudiado (Figura 3.1) fue comparado con ejemplares vivientes y fósiles de Conepatus. Se midieron cráneos de especies actuales en colecciones de Chile (C. chinga y C. humboldtii), Venezuela ( $C$. semistriatus) y Argentina (C. chinga, C. humboldtii y C. semistriatus) y de Austria (C. chinga). Se tomaron dos medidas craneanas: ACP, Ancho Constricción Postorbital y ACC, Altura de la Caja Craneana, excluyendo el "abultamiento mastoideo". Dado que el fósil estudiado es muy fragmentario, se utilizó el ACC ya que esta medida no poseía puntos craneométricos ausentes. En Argentina se obtuvieron el ACC y ACP del ejemplar MLP-1068 (C. mercedensis Reig, 1952) y el ACP de la fotografía del ejemplar MPS1 (C. primaevus Burmeister, 1864) obtenida de Forasiepi (2003). Se obtuvo el ACP del ejemplar UCV-VF10032 (C. semistriatus) de Venezuela, desde los datos obtenidos por Bocquentin-Villanueva (1979). Las medidas se obtuvieron con un calibre digital, excepto en el ejemplar Conepatus primaevus MPS1 cuyas medidas se tomaron con el programa TpsDig. Se usó el programa STAGRAPHICS Plus (5.1) y se realizaron las pruebas de Contraste de Varianza, Contraste Múltiple de Rangos y Comparación entre Medias, para explorar la existencia de diferencias entre las especies vivientes $(C$. chinga, $C$. humboldtii y C. semistriatus). Se estudió la anatomía del encéfalo a través de imágenes obtenidas del equipo CT SCANNER SENSATION 16 COLLIMATTOR $2 \times 0.6 \mathrm{~mm}$ (marca Siemens) sobre un ejemplar de C. chinga (procedente de Argentina, depositado en LPUACh01) y sobre el ejemplar MHMOP/PI/34. Además, se confeccionó un endomolde de caucho siliconado del mismo ejemplar. Los resultados obtenidos se compararon con las descripciones encefálicas realizadas a los géneros de Mephitidae por otros autores (e.g. Bryant et al. 1993; Radinsky, 1973). La edad del ejemplar MHMOP/PI/34 fue obtenida a través de una interpolación lineal simple (basada en el modelo de edad construido para el sitio Pilauco). Se emplearon dos muestras datadas con radiocarbono, GEOUACH-15 (hueso, Pozna Radiocarbon Laboratory, Polonia) y GEOUACH-53 (madera, NSF Arizona AMS Facility), con edades radiocarbónicas de $12.540 \pm 90$ y $11.834 \pm 186$ años A.P., ubicadas 10 y $76 \mathrm{~cm}$ por debajo y encima del fósil, respectivamente. Las edades calibradas con el programa Calib 4.4.2 corresponden a 14,7 y $13,7 \mathrm{Ka}$, respectivamente.

\section{RESULTADOS}

\section{Paleontología sistemática}

Clase MAMMALIA Linnaeus, 1758

Orden CARNIVORA Bowdich, 1821

Suborden CANIFORMIA Kretzoi, 1938

Familia MEPHITIDAE Bonaparte, 1845

Subfamilia MEPHITINAE Bonaparte, 1845

Conepatus Gray, 1837

Especie tipo.

Conepatus humboldtii Gray, 1837.

Material referido.

MHMOP/PI/34: Gran parte de una caja craneana en la cual se conserva gran parte de la región parietal y temporal.

Procedencia geográfica.

El Sitio Paleontológico Pilauco, Osorno, centro-sur de Chile (40 $\left.36^{\circ} \mathrm{S}-73^{\circ} 07^{\prime} \mathrm{W}\right)$.

Procedencia, estratigrafía y edad.

El ejemplar MHMOP/PI/34 procede de la cuadrícula E11 a una profundidad de $416 \mathrm{~cm}$. El nivel portador (PB-9) corresponde al Pleistoceno tardío y el ejemplar de Pilauco tiene una edad de $13.9 \mathrm{Ka}$ (Piso/Edad Lujanense). La estratigrafía del sitio en esta cuadricula incluye una capa basal compuesta por una toba fina de color gris verdoso de edad equivalente a MIS5e, de espesor desconocido, sobre la cual se deposita una capa de turba color chocolate (PB-7; espesor $120 \mathrm{~cm}$ ) que incluye clastos dispersos de grava y los huesos de los fósiles de mamíferos, aves, insectos y vegetales diversos. Sobre ella se dispone una capa muy similar a la anterior que no contiene fósiles macrofósiles (PB-8; espesor $100 \mathrm{~cm}$ ). La capa superior corresponde a una turba pardo negra, muy pura, que contiene lentes de sedimentos fluviales (PB-9, espesor $120 \mathrm{~cm}$ ). 

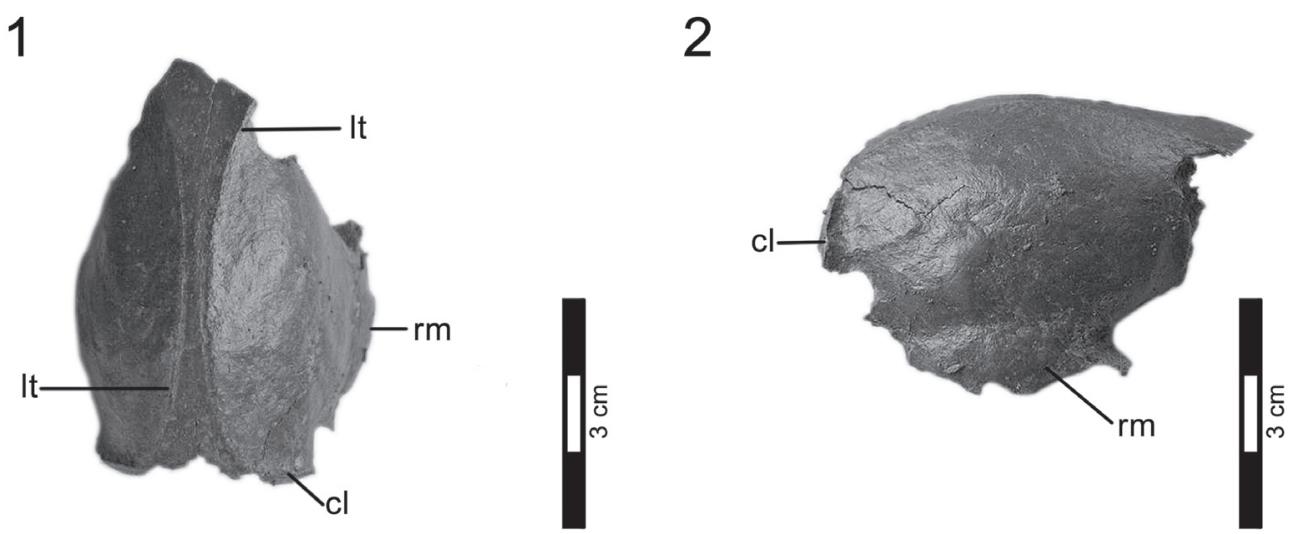

Fig. 3. Fotografías de la caja craneana de Conepatus sp. MHMOP/PI/34, 1. Vista dorsal, 2. Vista lateral derecha. Abreviaturas: cl, cresta lambdoidea; lt, líneas temporales; rm, región mastoidea.

Descripción.

La caja craneana es relativamente grande en tamaño en relación a los ejemplares muestreados y a los promedios de C. chinga informados por Van Gelder (1968). Se conserva gran parte de la caja craneana, pero faltan la región facial y palatal. No posee mandíbula, arcos cigomáticos ni bulas timpánicas. Posee forma de cúpula y las suturas están obliteradas. No presenta cresta sagital desarrollada, sino dos líneas temporales que van de la región frontal con dirección posterior hasta la región occipital. Estas líneas delimitan un área central que se comprime lateralmente en la mitad de la caja craneana formando un área liriforme (Figura 3.1). Luego de una breve compresión dichas líneas divergen en el extremo posterior de la caja craneana y confluyen con las crestas lambdoideas. En vista occipital, las crestas lambdoideas, forman una especie de "V" invertida (Forasiepi, 2003). Cada parietal articula posteriormente con el occipital formando las crestas lambdoideas y centralmente con los escamosos y rostralmente con los frontales. Las crestas lambdoideas constituyen el límite caudal del ejemplar aquí descrito, dado que la región occipital está ausente. El parietal forma la mayor parte de la pared lateral de la caja craneana y sobre la cara lateral derecha se observa un abultamiento del cráneo dorsal al proceso mastoideo (Figura 3.2). Esta corresponde a una cavidad interna generada por parte de la invasión de la región mastoidea por la cavidad del oído medio (Bryant et al. 1993), es decir, por detrás del arco cigomático y por encima del proceso mastoideo, producida por la neumatización de la región mastoidea. En la parte más anterior del abultamiento preserva parte de la base del arco cigomático. En la cara lateral izquierda este abultamiento no se preservó. Destacamos la ausencia del tentorio óseo en la porción ventral del temporal. De las descripciones basadas en el escáner, es posible reconocer un corto surco silviano, el suprasilviano y surco lateral pobremente curvados. El postsilviano está poco desarrollado. El surco

Tabla 1. Análisis estadístico de los valores de las medidas de la muestra de las especies sudamericanas vivientes y fósiles.

\begin{tabular}{|c|c|c|c|c|c|c|c|c|}
\hline C. humboldtii & $\mathrm{N}$ & $\mathrm{X}$ & $\mathrm{S}$ & $\mathrm{DE}$ & $\mathrm{EE}$ & $\mathrm{CV}$ & MAX-MIN & RANGO \\
\hline $\mathrm{ACP}$ & 22 & 16,54 & 0,59 & 0,77 & 0,16 & 0,04 & $18,21-15,48$ & 2,73 \\
\hline $\mathrm{ACC}$ & 22 & 19,17 & 16,53 & 4,06 & 0,86 & 0,21 & $24,11-12,17$ & 11,41 \\
\hline & & & & & & & & \\
\hline C. chinga & $\mathrm{N}$ & $\mathrm{X}$ & $\mathrm{S}$ & $\mathrm{DE}$ & $\mathrm{EE}$ & $\mathrm{CV}$ & $\mathrm{MAX}-\mathrm{MIN}$ & RANGO \\
\hline $\mathrm{ACP}$ & 65 & 17,76 & 4,00 & 2,00 & 0,24 & 0,03 & $23,12-14,12$ & 9,00 \\
\hline $\mathrm{ACC}$ & 65 & 22,81 & 2,85 & 1,68 & 0,20 & 0,07 & $26,93-19,28$ & 7,65 \\
\hline & & & & & & & & \\
\hline C. semistriatus & $\mathrm{N}$ & $\mathrm{X}$ & $\mathrm{S}$ & $\mathrm{DE}$ & $\mathrm{EE}$ & $\mathrm{CV}$ & MAX-MIN & RANGO \\
\hline $\mathrm{ACP}$ & 11 & 20,62 & 1,83 & 1,35 & 0,40 & 0,06 & $23,00-18,80$ & 4,20 \\
\hline $\mathrm{ACC}$ & 10 & 21,70 & 2,64 & 1,62 & 0,51 & 0,07 & $25,01-19,92$ & 5,09 \\
\hline
\end{tabular}


Tabla 2. Comparación de los valores más altos de las medidas de la muestra de las especies sudamericanas vivientes y fósiles, comparada con el ejemplar MHMOP/PI/34. Abreviaturas: (f) fósil; s/r sin registro.

\begin{tabular}{|c|c|c|c|c|}
\hline C. chinga & Valores & Edad & Sexo & Procedencia \\
\hline $\mathrm{ACP}$ & 22,60 & \multirow{2}{*}{ Adulto } & \multirow{2}{*}{ Indeterminado } & \multirow{2}{*}{$\begin{array}{l}\text { Valparaíso } \\
\text { Chile } \\
\text { NMW2474 }\end{array}$} \\
\hline ACC & 26,27 & & & \\
\hline \multicolumn{5}{|c|}{ C. chinga } \\
\hline $\mathrm{ACP}$ & 22,20 & \multirow{2}{*}{ Adulto } & \multirow{2}{*}{ Indeterminado } & \multirow{2}{*}{$\begin{array}{c}\text { Llanquihue } \\
\text { Chile } \\
\text { NMW1052 }\end{array}$} \\
\hline $\mathrm{ACC}$ & 25,30 & & & \\
\hline \multicolumn{5}{|c|}{ C. semistratus } \\
\hline $\mathrm{ACP}$ & 19,74 & \multirow{2}{*}{ Adulto } & \multirow{2}{*}{ Hembra } & \multirow{2}{*}{$\begin{array}{l}\text { Ecuador } \\
\text { MLP } 3162\end{array}$} \\
\hline ACC & 25,01 & & & \\
\hline \multicolumn{5}{|c|}{ C. chinga } \\
\hline $\mathrm{ACP}$ & 19,67 & \multirow{2}{*}{ Adulto } & \multirow{2}{*}{ Macho } & \multirow{2}{*}{$\begin{array}{l}\text { Tucumán } \\
\text { Argentina } \\
\text { IML6314 }\end{array}$} \\
\hline ACC & 26,93 & & & \\
\hline \multicolumn{5}{|c|}{ C. primaevus (f) } \\
\hline $\mathrm{ACP}$ & 21,63 & \multirow{2}{*}{ Adulto } & \multirow{2}{*}{ Indeterminado } & \multirow{2}{*}{$\begin{array}{c}\text { Buenos Aires } \\
\text { Argentina } \\
\text { MACNPv2444 }\end{array}$} \\
\hline ACC & $\mathrm{s} / \mathrm{r}$ & & & \\
\hline \multicolumn{5}{|c|}{ C. semistratus $(\mathrm{f})$} \\
\hline $\mathrm{ACP}$ & 23,00 & \multirow{2}{*}{ Adulto } & \multirow{2}{*}{ Indeterminado } & \multirow{2}{*}{$\begin{array}{c}\text { Muaco } \\
\text { Venezuela } \\
\text { UCVVF10032 }\end{array}$} \\
\hline ACC & $\mathrm{s} / \mathrm{r}$ & & & \\
\hline \multicolumn{5}{|c|}{ C. mercedensis $(\mathrm{f})$} \\
\hline $\mathrm{ACP}$ & 17,00 & \multirow{2}{*}{ Adulto } & \multirow{2}{*}{ Indeterminado } & \multirow{2}{*}{$\begin{array}{c}\text { Buenos Aires } \\
\text { Argentina } \\
\text { MLP1068 }\end{array}$} \\
\hline ACC & 22,00 & & & \\
\hline \multicolumn{5}{|c|}{ Conepatus sp. (f) } \\
\hline $\mathrm{ACP}$ & 20,06 & \multirow{2}{*}{ Subadulto } & \multirow{2}{*}{ Indeterminado } & \multirow{2}{*}{$\begin{array}{c}\text { Pilauco } \\
\text { Chile } \\
\text { MHMOP/PI/34 }\end{array}$} \\
\hline ACC & 25,60 & & & \\
\hline
\end{tabular}

postlateral está bien desarrollado en Conepatus de acuerdo a nuestros análisis. También observamos la presencia del surco cruciado.

\section{ANÁLISIS CUANTITATIVO}

Las medidas craneanas del nuevo resto fósil de Conepatus son ACP = 20,06 mm y ACC $=25,60 \mathrm{~mm}$ (Tabla 1). Los análisis estadísticos realizados con las especies vivientes permitieron observar que el ACC presenta diferencias significativas en las desviaciones estándares de las tres especies sudamericanas, pero se forma un grupo homogéneo entre C. semistriatus y $C$. humboldtii, existiendo un solapamiento entre ambas especies con respecto de las medias. El ACP presentó diferencias en las desviaciones estándares de las tres especies y no se formaron grupos homogéneos cuando se compararon las medias. El valor del ACC del MHMOP/PI/34 se encuentra únicamente dentro del rango de C. chinga, aunque es un 10,9\% mayor que la media de esta especie. El valor del ACP del MHMOP/PI/34 es casi idéntico a la media de C. semistriatus y se aleja de las medias de C. chinga y C. humboldtii en un $11,5 \%$ y $17,5 \%$ respectivamente. El ACP del ejemplar MHMOP/ $\mathrm{PI} / 34$ se encuentra dentro del rango de C. chinga y C. semistristus (Tabla 2).

\section{DISCUSIÓN}

La descripción realizada al ejemplar MHMOP/ $\mathrm{PI} / 34$ nos permite concluir que corresponde a un individuo subadulto. Tal condición es observada 
en individuos de las especies de C. leuconotus Lichtenstein, 1832, C. chinga y C. semistristus. El ejemplar MHMOP/PI/34 presenta la invasión de la región mastoidea por la cavidad del oído medio, lo que queda representado por una convexidad sobre la cara externa de la caja craneana por encima del proceso mastoideo (Bryant et al. 1993). En la mayoría de los caniformes el contorno craneano desarrolla una superficie cóncava inmediatamente por encima del meato auditivo externo y del proceso mastoideo (Bryant et al. 1993). El tentorio óseo está bien desarrollado en la mayoría de los carnívoros (Bryant et al. 1993), sin embargo, en los Mephitidae se encuentra muy reducido o ausente. Estos son rasgos diagnósticos de la familia Mephitidae y están presentes en el ejemplar MHMOP/PI/34.

En Conepatus los parietales están posteriormente expandidos, generando una caja craneana con apariencia abultada (Martin, 1978), característica observada en el fósil aquí descrito. En Mephitis, en vista lateral y a partir del bregma, la caja craneana es deprimida en dirección posterior, algo similar a lo observado en Spilogale Gray, 1865 en donde

1

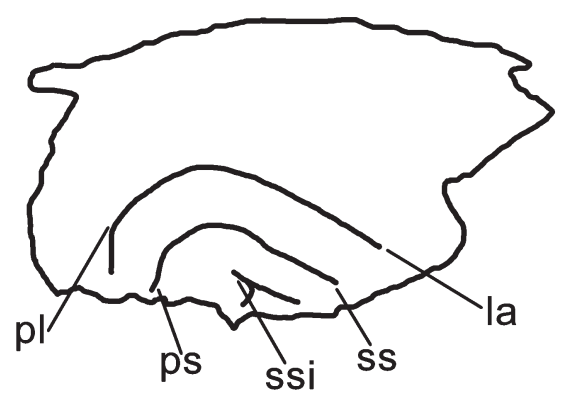

3

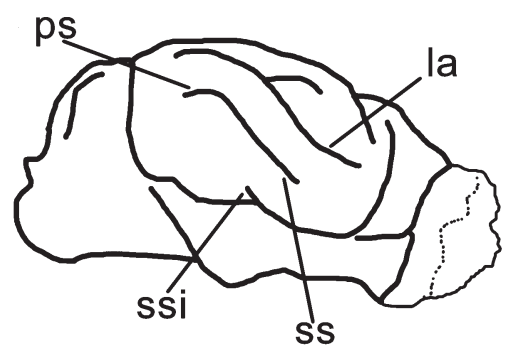

la región parietal y frontal están a la misma altura (Hall, 1936). Conepatus se diferencia de Mephitis y Spilogale por presentar una cresta lambdoidea menos desarrollada con respecto a la caja craneana. El contorno de la cresta lambdoidea, en el caso de Conepatus forma aproximadamente un ángulo recto, mientras que en Spilogale y Mephitis es más bien un ángulo obtuso con respecto al proceso mastoideo. Las condiciones observadas en Conepatus están presentes en el fósil estudiado, por lo cual podemos asignarlo a dicho género. En los hemisferios cerebrales de Conepatus se observan pliegues de bajorrelieve tales como un surco silviano corto, surco postsilviano y surco postlateral poco desarrollado (Radinsky, 1973), en comparación con los mustélidos. En el ejemplar MHMOP/PI/34 observamos un surco silviano corto, el suprasilviano y surco lateral pobremente curvados con respecto al rinal. El surco postsilviano está ausente o poco desarrollado en Conepatus (Radinsky, 1973), algo que también comparte el ejemplar MHMOP/PI/34. Sin embargo, hay diferencias entre los géneros de Mephitidae (incluido Mydaus Cuvier, 1821).
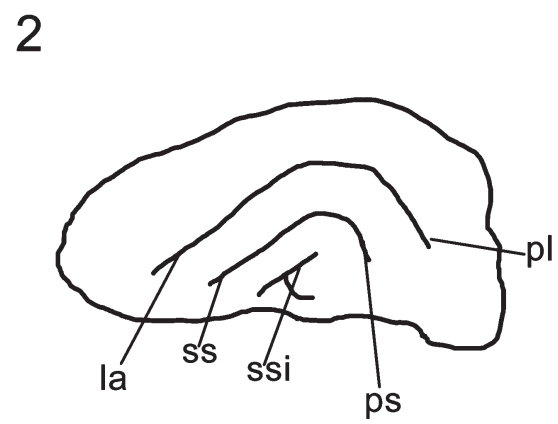

4

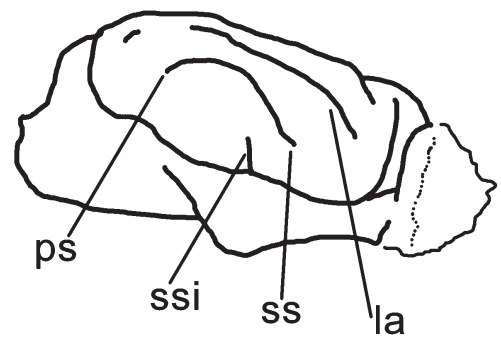

Fig. 4.1 Representación gráfica del escaneado de la vista ventral del fósil de Pilauco MHMOP/PI/34, 2. Representación gráfica del escaneado de la vista ventral del ejemplar actual de C. chinga. Representación gráfica de una comparación entre endomoldes (modificado de Radinsky, 1973), 3. Mephitis, 4. Spilogale. Abreviaturas: ssi, surco silviano; ss, surco suprasilviano; la, surco lateral; pl, surco postlateral; ps, surco postsilviano. 
Conepatus posee el surco suprasilviano y lateral más curvados, con mejor desarrollo de surcos postsilvianos y postlaterales (Radinsky, 1973) con respecto a los otros géneros de Mephitidae. En las imágenes obtenidas del surco postlateral mediante el escáner, tanto el actual Conepatus chinga como el ejemplar MHMOP/PI/34 muestran el surco postlateral bien desarrollado y levemente más cercano al surco rinal, que lo observado y figurado para Conepatus semistriatus por Radinsky (1973) (Figuras 4.1-2). En Mephitis los surcos postlateral y postsilviano están muy poco desarrollados y lateralmente constreñidos (Figura 4.3). Spilogale (Figura 4.4), muestra mejor desarrollo del surco postlateral y postsilviano que en los ejemplares más grandes de Mephitis (Radinsky, 1973). En la región de altorrelieve del cerebro, el molde endocraniano del ejemplar $\mathrm{MHMOP} / \mathrm{PI} / 34$ mostró que la disposición del surco cruciado forma un ángulo más agudo y cercano al surco longitudinal y es similar a la descripción que hace Radinsky (1973) de Conepatus semistriatus. En Spilogale y Mephitis, la disposición del surco cruciado es relativamente más perpendicular con respecto al surco longitudinal (Radinsky, 1973) y el surco cruciado de C. semistriatus es similar al de Spilogale, pero se diferencia de éste en que el surco cruciado es levemente más curvado (Figuras 5.1-2-3-4-5).

El análisis cuantitativo (Tabla 2) permitió determinar que las dimensiones del ejemplar MHMOP/ PI/34 están dentro del rango de tamaño de la especie $C$. chinga, así también, con algunos valores en la medida del ACC de individuos de C. chinga provenientes de las localidades de Valparaíso

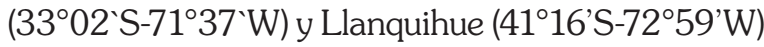
(ejemplares NMW2474 y NMW1052 respectivamente). Por otra parte, la latitud desde la cual fue recuperado el ejemplar $\mathrm{MHMOP} / \mathrm{PI} / 34$ coincide con la distribución actual de esta especie en Chile. Sin embargo, de nuestro análisis no resultan evidencias significativas para afirmar que el ejemplar MHMOP/PI/34 corresponda a la especie C. chinga. Por otra parte, dado lo fragmentario del ejemplar $\mathrm{MHMOP} / \mathrm{PI} / 34$, no se detectan rasgos anatómicos para separarlo de otras especies extinguidas de gran tamaño como C. primaevus. Algunos ejemplares de la especie $C$. chinga provenientes del noroeste de Argentina (provincia de Tucumán) y algunos ejemplares de Valparaíso (Chile) son en tamaño
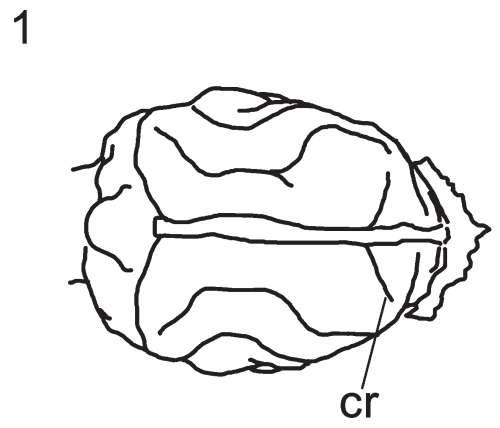

2

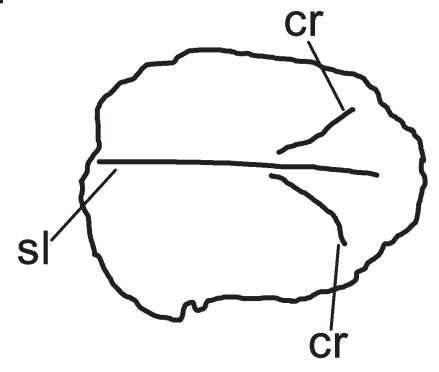

3

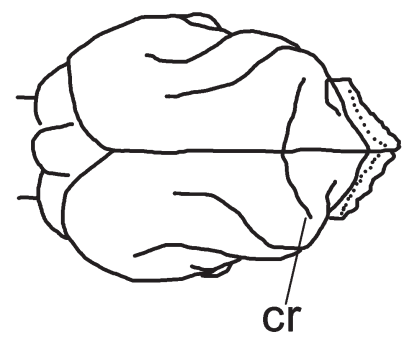

4
5

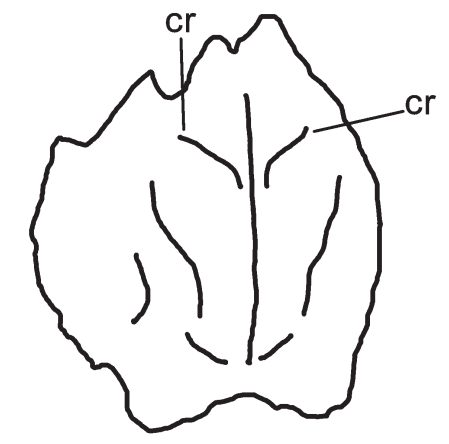

Fig. 5. Comparación del surco cruciado entre las especies americanas y el fósil de Pilauco, MHMOP/PI/34,

1. Conepatus semistriatus, 2. Representación gráfica del endomolde del fósil de Pilauco, MHMOP/PI/34, 3. Spilogale putorius, 4. Mephitis mephitis, 5. Representación gráfica del escaneado del fósil de Pilauco, MHMOP/

PI/34 (1, 3 y 4 modificados de Radinsky, 1973). Abreviaturas: cr, surco cruciado; sl, surco longitudinal. 
similares al ejemplar MHMOP/PI/34. Si comparamos el ejemplar MHMOP/PI/34 con la especie C. talarae Churcher y Zyll de Jong, 1965 (ejemplar fósil registrado en el Pleistoceno tardío de Perú) ésta especie representa una forma pequeña cercana al tamaño de la actual especie C. humboldtii (Wang y Carranza-Castañeda, 2008). C. mercedensis es la más pequeña de las especies analizadas en la muestra. Con respecto a la especie C. primaevus, el ACP no presenta diferencias significativas con respecto al ejemplar $\mathrm{MHMOP} / \mathrm{PI} / 34$. Estadísticamente significativa es la diferencia con el fósil de la especie $C$. semistriatus, ya que con respecto al $\mathrm{ACP}$, el ejemplar MHMOP/PI/34 es mas pequeño y se aleja en un $14,7 \%$. Este fósil de Venezuela es superado levemente sólo por un ejemplar adulto y actual de la especie $C$. chinga (ACP 23,12 mm; 7150 IML) de Tucumán, Argentina. Si bien existen algunas diferencias entre las especies sudamericanas del género Conepatus, por lo discutido más arriba, no es posible determinar al ejemplar fósil de Chile a nivel específico.

Al final del siglo XIX Roth (1899) asignó un material mandibular a la especie Conepatus suffocans (actualmente sinónimo de C. chinga para las poblaciones distribuidas en Argentina y Brasil) IIliger, 1811 para la Cueva del Milodón $\left(51^{\circ} 36^{\prime} \mathrm{S}-72^{\circ} 36^{\prime} \mathrm{W}\right.$; XII Región de Chile), sin embargo, los restos fueron referidos posteriormente a Lyncodon patagonicus de Blainville, 1842 por Smith-Woodward (1900), y posteriormente asignados a Galictis sp. Bell, 1826 por Prevosti y Pardiñas (2001). Por otro lado, Borrero et al. (1997) mencionaron un ejemplar de C. humboldtii para La Cueva Lago Sofía 4 (513'ㅇ7240`W; XII Región, Chile), el que tendría una edad más moderna asociada a ocupaciones del Holoceno tardío. Estas citas fueron las empleadas por Frassinetti y Alberdi (2001) para indicar la presencia de méfitidos en el Pleistoceno tardío de Chile. Esta ausencia de registros para el Pleistoceno ya había sido notada por Latorre (1998), quien no incluye al género Conepatus entre los carnívoros hallados en Chile para el Pleistoceno dado que los ejemplares de la cueva del Lago Sofía resultaron ser sub-recientes. Por lo tanto, de acuerdo a estos antecedentes, el ejemplar MHMOP/PI/34 corresponde al primer registro confirmado y descrito de Mephitidae del Pleistoceno para Chile, amplía la paleodistribución en Sudamérica y constituye el méfitido pleistocénico más austral, ya que los registros anteriores están circunscritos hasta los $38^{\circ} \mathrm{S}$.

\section{AGRADECIMIENTOS}

A L. Pomi e I. Olivares, a D. Flores y A. Kramarz, a E. Chávez, J. Cárcamo, F. Mondaca, B. Herzig y A. Bilb por la ayuda brindada durante el estudio de sus respectivas colecciones. A la Clínica Alemana de Valdivia y al Fondo Nacional de Desarrollo Regional de Chile, Región de los Lagos, proyecto FNDR 2308-42-LE07. La contribución de M. Pino y E. González fue parcialmente financiada por el proyecto FONDECYT 1100555.

\section{BIBLIOGRAFÍA}

AMEGHINO, F. 1889. Contribución al conocimiento de los mamíferos fósiles de la República Argentina. Academia Nacional de Ciencias de Córdoba, Actas, Vol. 6, 1027 pp. Córdoba, Argentina.

BERMAN, W. 1994. Los carnívoros continentales (Mammalia, Carnívora) del Cenozoico en la provincia de Buenos Aires. Tesis Doctoral, Facultad de Ciencias Naturales y Museo, Universidad Nacional de La Plata (Inédito).

BOCQUENTIN-VILLANUEVA, J. 1979. Mammiferes fósiles du Pleistocene superieur de Muaco, Etat de Falcón, Venezuela. Tesis Doctoral inédita. L'Université Pierre et Marie Curie. París.

BORRERO, L. A., MARTIN F. M. y PRIETO A. 1997. La Cueva Lago Sofía 4. Una madriguera Pleistocénica. Anales del Instituto de la Patagonia, Serie Ciencias Sociales 25:103-122, Punta Arenas.

BRYANT, H.N., RUSSELL, A.P. y FITCH, W.D. 1993. Phylogenetic relationships within the extant Mustelidae (Carnivora): appraisal of the cladistic status of the Simpsonian subfamilies. Zoological Journal of the Linnean Society 108: 301-334.

CHURCHER, C. y VAN ZYLL DE JONG 1965. Conepatus talarae n sp. From the Talara Tar-seeps, Perú. Life Sciences Contribution 62: 1-15.

DRAGOO, J. y HONEYCUTT, R. 1997. Systematics of mustelidlike carnivores. Journal of Mammalogy, 78(2): 426-443.

DRAGOO, J., HONEYCUTT, R. y SHMIDLY, D. 2003. Taxonomic status of white-backed hog- nosed skunks, genus Conepatus (Carnivora: Mephitidae) Journal of Mammalogy, 84(1):159-176.

FLYNN, J., FINARELLI, J., ZEHR, S., HSU, J. y NEDBAL, M. 2005a. Molecular phylogeny of the Carnivora (Mam- 
malia): assessing the impact of increased sampling on resolving enigmatic relationships. Systematic Biology, 54(2): 317-337.

FORASIEPI, A. 2003. Nuevo registro de Conepatus primaevus (Mammalia, Carnívora, Mustelidae) del Pleistoceno de la Provincia de Buenos Aires. Revista Museo Argentino Ciencias Naturales, n.s, 5(1):21-29.

FRASSINETTI, D. y ALBERDI, M. 2001. Los macromamíferos continentales del Pleistoceno Superior de Chile: Reseña histórica, localidades, restos fósiles, especies y dataciones. Estudios Geológicos 57(1-2).

FULLER, T., JOHNSON, W., FRANKLIN, W. y JOHNSON, K. 1987. Notes on the Patagonian hog-nosed skunk (Conepatus humboldti) in southern Chile. Journal of Mammalogy 68: 864-867.

HALL, E. R. 1936. Mustelid mammals from the Pleistocene of North America with systematic notes on some recent members of the genera Mustela, Taxidea, and Mephitis. Carnegie Institution of Washington Publication 473:41-119.

HOFFSTETTER, R. 1963. La faune Pleistocene de Tarija (Bolivie), note preliminaire. Bulletin Muséum National d Histoire Naturelle, 35(2): 194-203.

LATORRE, C. 1998. Paleontología de mamíferos del Alero Tres Arroyos 1, Tierra del Fuego, XII Región, Chile. Anales del Instituto de la Patagonia, Serie Ciencias Naturales, 26: 77-90. Punta Arenas.

MARTIN, R. 1978. An new late Pleistocene Conepatus and associated vertebrate fauna from Florida. Journal of Paleontology. 52(5):1079-1085.

PARERA, A., 2002. Los mamíferos de la Argentina y la región austral de Sudamérica. Edición. El Ateneo, Buenos Aires (Primera Edición). 453 pp.

PAULA COUTO, J.C. 1979. Tratado de Paleomastozoología. Academia Brasileira de Ciencias, 590 pp. Río de Janeiro.

PREVOSTI, F. y PARDIÑAS, U. 2001. Variaciones corológicas de Lyncodon patagonicus (Carnívora, Mustelidae) durante el Cuaternario. Mastozoología Neotropical, 8(1): 21-39.

QUINTANA, V. YÁÑEZ, J., y VALDEBENITO, M. 2000. Orden Carnívora. En: Mamíferos de Chile. Ediciones Centros Estudios Ambientales, Valdivia Chile. 161-162 p.
RADINSKY, L. 1973. Are stink badgers skunks? Implications of neuroanatomy for mustelid phylogeny. Journal of Mammalogy 54:585-593.

REIG, O. 1952. Sobre la presencia de mustélidos mefitinos en la Formación Chapadmalal. Revista del Museo Municipal de Ciencias Naturales de Mar del Plata, (1):45-51.

ROTH, S. 1899. El mamífero misterioso de la Patagonia Grypotherium domesticum. 2. Descripción de los restos encontrados en la Caverna de Ultima Esperanza. Revista del Museo de La Plata (Argentina) 9: 421-453.

SATO, J., HOSADA T., WOLSAN, M. y SUZUKI H. 2004. Molecular phylogeny of arctoids (Mammalia: Carnivora) with emphasis on phylogenetic and taxonomic positions of the ferret-badgers and skunks. Zoologial Science, 21: 111-118.

SMITH-WOODWARD, A. 1900. On some remains of Grypotherium (Neomylodon) listai and associated mammals from a cavern near Consuelo Cave, Last Hope Inlet, Patagonia, Proceeding Zoological Society London: 64-79.

SOIBELZON, L. y PREVOSTI, F. 2007. Los carnívoros (Carnivora, Mammalia) terrestres del Cuaternario de América del Sur En: Geomorfologia Litoral i Quaternari. Homenatge a Joan Cuerda Barceló ed. Palma de mallorca : Monografies de la Societat d'Historia Natural de les Balears.

VAN GELDER, R. 1968. The Genus Conepatus (Mammalia, Mustelidae): Variation Within a Population. American Museum Novitates 2322:1-37.

WANG, X. y CARRANZA-CASTAÑEDA O. 2008. Earliest hog-nosed skunk, Conepatus (Mephitidae, Carnivora), from the early Pliocene of Guanajuato, Mexico and origin of South American skunks. Zoological Jorunal of the Linnean Society, 154, 386-407.

WOODBURNE, M., CIONE A.L. y TONNI, E.P. 2006. Central American Provincialism and the Great American Biotic Interchange. En O. Carranza-Castañeda y E.H. Lindsay (editions.), Advances in late Tertiary vertebrate paleontology in Mexico and the Great American Biotic Interchange. Publicación Especial del Instituto de Geología y Centro de Geociencias de la Universidad Nacional Autónoma de México 4: 73-101. 